\title{
Weakly nonassociative algebras, Riccati and KP hierarchies*
}

\author{
Aristophanes Dimakis \\ Department of Financial and Management Engineering, \\ University of the Aegean, 31 Fostini Str., GR-82100 Chios, Greece \\ dimakis@aegean.gr \\ Folkert Müller-Hoissen \\ Max-Planck-Institute for Dynamics and Self-Organization \\ Bunsenstrasse 10, D-37073 Göttingen, Germany \\ folkert.mueller-hoissen@ds.mpg.de
}

\begin{abstract}
It has recently been observed that certain nonassociative algebras (called "weakly nonassociative", WNA) determine, via a universal hierarchy of ordinary differential equations, solutions of the KP hierarchy with dependent variable in an associative subalgebra (the middle nucleus). We recall central results and consider a class of WNA algebras for which the hierarchy of ODEs reduces to a matrix Riccati hierarchy, which can be easily solved in certain cases. The resulting solutions of a matrix KP hierarchy then determine (under a rank 1 condition) solutions of the scalar KP hierarchy. We also extend these results to the discrete KP hierarchy. Moreover, we build a bridge from the WNA framework to the Gelfand-Dickey formulation of the KP hierarchy.
\end{abstract}

\section{Introduction}

The Kadomtsev-Petviashvili (KP) equation is an extension of the famous Korteweg-deVries (KdV) equation to $2+1$ dimensions. It first appeared in plasma physics [1,2], in the context of the stability problem of $\mathrm{KdV}$ solitons. It also describes nonlinear fluid surface waves in a certain approximation and explains to some extent the formation of structures formed by water waves [2]. It is "integrable" in several respects, in particular in the sense of the inverse scattering method. Various remarkable properties have been discovered which allow to access (subsets of) its solutions in different ways, see in particular [3-5]. Apart from its direct relevance in physics, the KP equation and its hierarchy (see [5,6], for example) is deeply related to the theory of Riemann surfaces (Riemann-Schottky problem, see [7] for a review). Some time ago, this induced discussions concerning the role of KP in string theory (see [8-11], for example). Later the Gelfand-Dickey hierarchies, of which the KdV hierarchy is the simplest and which are reductions of the KP hierarchy, made their appearance in matrix models, first in a model of two-dimensional quantum gravity (see $[12,13]$ and references therein). This led to important developments in algebraic geometry (see [14], for example). Of course, what we mentioned here by far does not exhaust what is known about $\mathrm{KP}$ and there is probably even much more in the world of mathematics and physics linked to the KP equation and its descendants that still waits to be uncovered.

In fact, an apparently completely different appearance of the KP hierarchy has been observed in [15]. Under certain conditions, there is a simple construction of a family of commuting derivations on a class

*(C)2007 by A. Dimakis and F. Müller-Hoissen 
of "weakly nonassociative" (WNA) algebras (see section 2).1. It turns out that they satisfy identities which are in an algebraic correspondence with the equations of the KP hierarchy. As a consequence, there is a hierarchy of ordinary differential equations (ODEs) on such nonassociative algebras which imply the KP hierarchy (for a dependent variable which lives in an associative subalgebra). In this way WNA algebras determine classes of solutions of the KP hierarchy.

In section 2 we recall central results of [15] and also present a formula in the WNA framework which is related to Bäcklund (or Darboux) transformations of the KP hierarchy. Section 3 applies the WNA approach to derive a matrix Riccat 2 hierarchy, the solutions of which are solutions of the corresponding matrix KP hierarchy (which under certain conditions determines solutions of the scalar KP hierarchy). In section 4 we extend these results to the discrete KP hierarchy [35-39]. Furthermore, in section 5 we show how the Gelfand-Dickey formulation [5] of the KP hierarchy (with dependent variable in any associative algebra) emerges in the WNA framework. Section 6 contains some conclusions.

\section{Nonassociativity and KP}

In [15] we called an algebra $(\mathbb{A}, \circ)$ (over a commutative ring) weakly nonassociative (WNA) if

$$
(a, b c, d)=0 \quad \forall a, b, c, d \in \mathbb{A},
$$

where $(a, b, c):=(a \circ b) \circ c-a \circ(b \circ c)$ is the associator in $\mathbb{A}$. The middle nucleus of $\mathbb{A}$ (see [40], for example),

$$
\mathbb{A}^{\prime}:=\{b \in \mathbb{A} \mid(a, b, c)=0 \quad \forall a, c \in \mathbb{A}\},
$$

is an associative subalgebra and a two-sided ideal. Let us fix $f \in \mathbb{A} \backslash \mathbb{A}^{\prime}$ and define $a \circ_{1} b:=a \circ b$ and

$$
a \circ_{n+1} b:=a \circ\left(f \circ_{n} b\right)-(a \circ f) \circ_{n} b \quad n=1,2, \ldots .
$$

As a consequence of $(2.1)$, these products only depend on the equivalence class $[f]$ of $f$ in $\mathbb{A} / \mathbb{A}^{\prime}$.

The subalgebra $\mathbb{A}(f)$, generated by $f$ in the WNA algebra $\mathbb{A}$, is called $\delta$-compatible if, for each $n \in \mathbb{N}$,

$$
\delta_{n}(f):=f \circ_{n} f
$$

extends to a derivation of $\mathbb{A}(f)$. In the following we recall some results from [15].

Theorem 1 Let $\mathbb{A}(f)$ be $\delta$-compatible. The derivations $\delta_{n}$ then commute on $\mathbb{A}(f)$ and satisfy identities which are in correspondence via

$$
\delta_{n} \mapsto \partial_{t_{n}}
$$

(where $\partial_{t_{n}}$ is the partial derivative operator with respect to a variable $t_{n}$ ) with the equations of the potential Kadomtsev-Petviashvili ( $p K P$ ) hierarchy with dependent variable in $\mathbb{A}^{\prime}$.

This is a central observation in [15] with the following immediate consequence.

\footnotetext{
${ }^{1}$ Families of commuting derivations on certain algebras also appeared in [16,17], for example. In fact, the ideas underlying the work in [15] grew out of our work in [18] which has some algebraic overlap with [16].

${ }^{2}$ Besides their appearance in control and systems theory, matrix Riccati equations (see [19-21], for example) frequently showed up in the context of integrable systems, see in particular [22-34].
} 
Theorem 2 Let $\mathbb{A}$ be any WNA algebra over the ring of complex functions of independent variables $t_{1}, t_{2}, \ldots$. If $f \in \mathbb{A}$ solves the hierarchy of ODE $\sqrt{3}$

$$
f_{t_{n}}=f \circ_{n} f \quad n=1,2, \ldots,
$$

then $-f_{t_{1}}$ lies in $\mathbb{A}^{\prime}$ and solves the KP hierarchy with dependent variable in $\mathbb{A}^{\prime}$.

Corollary 1 If there is a constant $\nu \in \mathbb{A} \backslash \mathbb{A}^{\prime}$ with $[\nu]=[f] \in \mathbb{A} / \mathbb{A}^{\prime}$, then, under the assumptions of theorem 2

$$
\phi:=\nu-f \in \mathbb{A}^{\prime}
$$

solves the potential KP hierarchy 4

$$
\sum_{i, j, k=1}^{3} \varepsilon_{i j k}\left(\lambda_{i}^{-1}\left(\phi_{\left[\lambda_{i}\right]}-\phi\right)+\phi \circ \phi_{\left[\lambda_{i}\right]}\right)_{\left[\lambda_{k}\right]}=0,
$$

where $\varepsilon_{i j k}$ is totally antisymmetric with $\varepsilon_{123}=1, \lambda_{i}, i=1,2,3$, are indeterminates, and $\phi_{ \pm[\lambda]}(\mathbf{t}):=$ $\phi(\mathbf{t} \pm[\lambda])$, where $\mathbf{t}=\left(t_{1}, t_{2}, \ldots\right)$ and $[\lambda]:=\left(\lambda, \lambda^{2} / 2, \lambda^{3} / 3, \ldots\right)$.

Remark 1. If $C \in \mathbb{A}^{\prime}$ is constant, then $f=\nu^{\prime}-(\phi+C)$ with constant $\nu^{\prime}:=\nu+C$ satisfying $\left[\nu^{\prime}\right]=[\nu]=[f]$. Hence, with $\phi$ also $\phi+C$ is a solution of the pKP hierarchy. This can also be checked directly using (2.7), of course.

The next proposition is related to Bäcklund transformations of the KP hierarchy and will be used in section 4

Proposition 1 Suppose $f$ and $f^{\prime}$ solve $(2.5)$ and $[f]=\left[f^{\prime}\right]$ in a WNA algebra $\mathbb{A}$. The equation

$$
f^{\prime} \circ f=\alpha\left(f^{\prime}-f\right)
$$

is then preserved for all $\alpha \in \mathbb{C}$.

Proof:

$$
\begin{aligned}
\left(f^{\prime} \circ f\right)_{t_{n}}= & f_{t_{n}}^{\prime} \circ f+f^{\prime} \circ f_{t_{n}}=\left(f^{\prime} \circ_{n} f^{\prime}\right) \circ f+f^{\prime} \circ\left(f \circ_{n} f\right) \\
= & \left(f^{\prime} \circ_{n} f^{\prime} \circ \circ f-f^{\prime} \circ_{n}\left(f^{\prime} \circ f\right)+\alpha f^{\prime} \circ_{n}\left(f^{\prime}-f\right)\right. \\
& +f^{\prime} \circ\left(f \circ_{n} f\right)-\left(f^{\prime} \circ f\right) \circ_{n} f+\alpha\left(f^{\prime}-f\right) \circ_{n} f \\
= & -f^{\prime} \circ_{n+1} f+f^{\prime} \circ_{n+1} f+\alpha\left(f^{\prime} \circ_{n} f^{\prime}-f \circ_{n} f\right)=\alpha\left(f^{\prime}-f\right)_{t_{n}} .
\end{aligned}
$$

In the third step we have added terms which vanish as a consequence of (2.8). Then we used (3.11) in [15] (together with the fact that the products $\circ_{n}$ only depend on the equivalence class $[f]=\left[f^{\prime}\right] \in \mathbb{A} / \mathbb{A}^{\prime}$ ), and also (2.3), to combine pairs of terms into products of one degree higher.

Remark 2. In functional form, (2.5) can be expressed (e.g. with the help of results in [15]) as

$$
\lambda^{-1}\left(f-f_{-[\lambda]}\right)-f_{-[\lambda]} \circ f=0 .
$$

\footnotetext{
${ }^{3}$ We write $f_{t_{n}}$ for $\partial_{t_{n}}(f) . f$ has to be differentiable, of course, which requires a corresponding (e.g. Banach space) structure on $\mathbb{A}$. The flows given by [2.5) indeed commute [15]. Furthermore, 2.5) implies $\delta$-compatibility of the algebra $\mathbb{A}(f)$ generated by $f$ in $\mathbb{A}$ over $\mathbb{C}[15]$.

${ }^{4}$ This functional representation of the potential KP hierarchy appeared in [41,42]. See also [15,26] for equivalent formulae.
} 
Setting $f^{\prime}=f_{-[\lambda]}$ (which also solves (2.9) if $f$ solves it), this takes the form (2.8) with $\alpha=-\lambda^{-1}$.

In order to apply the above results, we need examples of WNA algebras. For our purposes, it is sufficient to recall from [15] that any WNA algebra with $\operatorname{dim}\left(\mathbb{A} / \mathbb{A}^{\prime}\right)=1$ is isomorphic to one determined by the following data:

(1) an associative algebra $\mathcal{A}$ (e.g. any matrix algebra)

(2) a fixed element $g \in \mathcal{A}$

(3) linear maps $\mathcal{L}, \mathcal{R}: \mathcal{A} \rightarrow \mathcal{A}$ such that

$$
[\mathcal{L}, \mathcal{R}]=0, \quad \mathcal{L}(a \circ b)=\mathcal{L}(a) \circ b, \quad \mathcal{R}(a \circ b)=a \circ \mathcal{R}(b) .
$$

Augmenting $\mathcal{A}$ with an element $f$ such that

$$
f \circ f:=g, \quad f \circ a:=\mathcal{L}(a), \quad a \circ f:=\mathcal{R}(a),
$$

leads to a WNA algebra $\mathbb{A}$ with $\mathbb{A}^{\prime}=\mathcal{A}$, provided that the following condition holds,

$$
\exists a, b \in \mathcal{A}: \mathcal{R}(a) \circ b \neq a \circ \mathcal{L}(b)
$$

This guarantees that the augmented algebra is not associative. Particular examples of $\mathcal{L}$ and $\mathcal{R}$ are given by multiplication from left, respectively right, by fixed elements of $\mathcal{A}$ (see the next section).

\section{A class of WNA algebras and a matrix Riccati hierarchy}

Let $\mathcal{M}(M, N)$ be the vector space of complex $M \times N$ matrices, depending smoothly on independent real variables $t_{1}, t_{2}, \ldots$, and let $S, L, R, Q$ be constant matrices of dimensions $M \times N, M \times M, N \times N$ and $N \times M$, respectively. Augmenting with a constant element $\nu$ and setting 5

$$
\nu \circ \nu=-S, \quad \nu \circ A=L A, \quad A \circ \nu=-A R, \quad A \circ B=A Q B,
$$

for all $A, B \in \mathcal{M}(M, N)$, we obtain a WNA algebra $(\mathbb{A}, \circ)$. The condition (2.12) requires

$$
R Q \neq Q L .
$$

For the products $\circ_{n}, n>1$, we have the following result.

\section{Proposition 2}

$$
\nu \circ_{n} \nu=-S_{n}, \quad \nu \circ_{n} A=L_{n} A, \quad A \circ_{n} \nu=-A R_{n}, \quad A \circ_{n} B=A Q_{n} B,
$$

where

$$
\left(\begin{array}{cc}
R_{n} & Q_{n} \\
S_{n} & L_{n}
\end{array}\right)=H^{n} \quad \text { with } \quad H:=\left(\begin{array}{cc}
R & Q \\
S & L
\end{array}\right)
$$

Proof: Using the definition (2.3), one proves by induction that

$$
\begin{aligned}
S_{n+1} & =L S_{n}+S R_{n}, & L_{n+1} & =L L_{n}+S Q_{n}, \\
R_{n+1} & =Q S_{n}+R R_{n}, & Q_{n+1} & =Q L_{n}+R Q_{n},
\end{aligned}
$$

\footnotetext{
${ }^{5}$ Using $(2.6$, in terms of $f$ this yields relations of the form 2.11).
} 
for $n=1,2, \ldots$, where $S_{1}=S, L_{1}=L, R_{1}=R, Q_{1}=Q$. This can be written as

$$
\left(\begin{array}{cc}
R_{n+1} & Q_{n+1} \\
S_{n+1} & L_{n+1}
\end{array}\right)=H\left(\begin{array}{cc}
R_{n} & Q_{n} \\
S_{n} & L_{n}
\end{array}\right)
$$

which implies (3.4).

Using (2.6) and (3.3) in (2.5), leads to the matrix Riccati equations 6

$$
\phi_{t_{n}}=S_{n}+L_{n} \phi-\phi R_{n}-\phi Q_{n} \phi \quad n=1,2, \ldots .
$$

Solutions of (3.5) are obtained in a well-known 'Grassmannian way' (see [21,32], for example) via

$$
\phi=Y X^{-1}
$$

from the linear system

$$
Z_{t_{n}}=H^{n} Z, \quad Z=\left(\begin{array}{c}
X \\
Y
\end{array}\right)
$$

with an $N \times N$ matrix $X$ and an $M \times N$ matrix $Y$, provided that $X$ is invertible. This system is obviously solved by

$$
Z(\mathbf{t})=e^{\xi(H)} Z_{0} \quad \text { where } \quad \xi(H):=\sum_{n \geq 1} t_{n} H^{n} .
$$

If $Q$ has rank 1, then

$$
\varphi:=\operatorname{tr}(Q \phi)
$$

defines a homomorphism from $(\mathcal{M}(M, N), \circ)$ into the scalars (with the ordinary product of functions). Hence, if $\phi$ solves the potential KP hierarchy in $(\mathcal{M}(M, N), \circ)$, then $\varphi$ solves the scalar pKP hierarchy 7 More generally, if $Q=V U^{T}$ with $V, U$ of dimensions $N \times r$, respectively $M \times r$, then $U^{T} \phi V$ solves the $r \times r$-matrix KP hierarchy.

Remark 3. If $\phi$ solves the pKP hierarchy, then also $\phi+C$ with a constant $M \times N$ matrix $C$ (see remark 1). This corresponds to the similarity transformation

$$
H \mapsto T H T^{-1} \quad \text { with } \quad T:=\left(\begin{array}{cc}
I_{N} & 0 \\
C & I_{M}
\end{array}\right)
$$

(where $I_{N}$ denotes the $N \times N$ unit matrix), which induces a transformation of equations (3.5).

Example 1. If $S=0$ and $Q=R K-K L$ with an $N \times M$ matrix $K$, then $Q_{n+1}+K L^{n+1}=$ $R\left(Q_{n}+K L^{n}\right)=R^{n}(Q+K L)=R^{n+1} K$. It follows that

$$
H^{n}=\left(\begin{array}{cc}
R^{n} & R^{n} K-K L^{n} \\
0 & L^{n}
\end{array}\right)
$$

\footnotetext{
${ }^{6}$ The corresponding functional form is $\lambda^{-1}\left(\phi_{[\lambda]}-\phi\right)+\phi Q \phi_{[\lambda]}=S+L \phi_{[\lambda]}-\phi R$, which is easily seen to imply (2.7), see also [43]. The appendix provides a FORM program [44,45] which independently verifies that any solution of $[3.5$, reduced to $n=1,2,3$, indeed solves the matrix pKP equation in $(\mathcal{M}(M, N), \circ)$.

${ }^{7}$ For related results and the significance of the rank 1 condition, see also [46] and the references cited there. The idea to look for (simple) solutions of matrix and more generally operator versions of an 'integrable' equation, and to generate from it (complicated) solutions of the scalar equation by use of a suitable map, already appeared in [47] and has been applied more recently in [48-54].
} 
and thus

$$
e^{\xi(H)}=\left(\begin{array}{cc}
e^{\xi(R)} & e^{\xi(R)} K-K e^{\xi(L)} \\
0 & e^{\xi(L)}
\end{array}\right) .
$$

If (3.2) holds, we obtain the following solution of the matrix pKP hierarchy in $(\mathcal{M}(M, N), \circ)$,

$$
\phi=e^{\xi(L)} \phi_{0}\left(I_{N}+K \phi_{0}-e^{-\xi(R)} K e^{\xi(L)} \phi_{0}\right)^{-1} e^{-\xi(R)},
$$

where $\phi_{0}=Y_{0} X_{0}^{-1}$. This in turn leads to

$$
\begin{aligned}
\varphi & =\operatorname{tr}\left(e^{-\xi(R)}(R K-K L) e^{\xi(L)} \phi_{0}\left(I_{N}+K \phi_{0}-e^{-\xi(R)} K e^{\xi(L)} \phi_{0}\right)^{-1}\right) \\
& =\operatorname{tr}\left(\log \left(I_{N}+K \phi_{0}-e^{-\xi(R)} K e^{\xi(L)} \phi_{0}\right)\right)_{t_{1}} \\
& =(\log \tau)_{t_{1}}, \quad \tau:=\operatorname{det}\left(I_{N}+K \phi_{0}-e^{-\xi(R)} K e^{\xi(L)} \phi_{0}\right) .
\end{aligned}
$$

If $\operatorname{rank}(Q)=1$, then $\varphi$ solves the scalar pKP hierarchy. Besides (3.2) and this rank condition, further conditions will have to be imposed on the (otherwise arbitrary) matrices $R, K, L$ and $\phi_{0}$ to guarantee that $\varphi$ is a real and regular solution. See [55], and references cited there, for classes of solutions obtained from an equivalent formula or restrictions of it. This includes multi-solitons and soliton resonances (KPII), and multi-lump solutions (after passing to KP-I via $t_{2 n} \mapsto \imath t_{2 n}$ and performing suitable limits of parameters).

Example 2. Let $M=N$ and

$$
L=S \pi_{-}, \quad R=\pi_{+} S, \quad Q=\pi_{+} S \pi_{-},
$$

with constant $N \times N$ matrices $\pi_{+}, \pi_{-}$subject to $\pi_{+}+\pi_{-}=I_{N}$. The matrix $H$ can then be written as

$$
H=\left(\begin{array}{c}
\pi_{+} \\
I_{N}
\end{array}\right) S\left(\begin{array}{ll}
I_{N} & \pi_{-}
\end{array}\right),
$$

which lets us easily calculate

$$
H^{n}=\left(\begin{array}{cc}
\pi_{+} S^{n} & \pi_{+} S^{n} \pi_{-} \\
S^{n} & S^{n} \pi_{-}
\end{array}\right)
$$

As a consequence, we obtain

$$
\phi=\left(-C_{+}+e^{\xi(S)} C_{-}\right)\left(\pi_{-} C_{+}+\pi_{+} e^{\xi(S)} C_{-}\right)^{-1},
$$

where $C_{ \pm}:=I_{N} \mp \pi_{ \pm} \phi_{0}$. This solves the matrix pKP hierarchy in $\mathcal{M}(M, N)$ with the product $A \circ B=$ $A \pi_{+} S \pi_{-} B$ if (3.2) holds, which is $\pi_{+} S\left(\pi_{+}-\pi_{-}\right) S \pi_{-} \neq 0$. If furthermore $\operatorname{rank}\left(\pi_{+} S \pi_{-}\right)=1$, then

$$
\varphi=\operatorname{tr}(Q \phi)=-\operatorname{tr}\left(\pi_{+} S\right)+(\log \tau)_{t_{1}}, \quad \tau=\operatorname{det}\left(\pi_{-} C_{+}+\pi_{+} e^{\xi(S)} C_{-}\right)
$$

solves the scalar pKP hierarchy (see also [43]). We will meet the basic structure underlying this example again in section 5 


\section{WNA algebras and solutions of the discrete KP hierarchy}

The potential discrete KP (pDKP) hierarchy in an associative algebra $(\mathcal{A}, \circ)$ can be expressed in functional form as follows 8

$$
\Omega(\lambda)^{+}-\Omega(\lambda)_{-[\mu]}=\Omega(\mu)^{+}-\Omega(\mu)_{-[\lambda]},
$$

where $\lambda, \mu$ are indeterminates,

$$
\Omega(\lambda):=\lambda^{-1}\left(\phi-\phi_{-[\lambda]}\right)-\left(\phi^{+}-\phi_{-[\lambda]}\right) \circ \phi,
$$

and $\phi=\left(\phi_{k}\right)_{k \in \mathbb{Z}}, \phi_{k}^{+}:=\phi_{k+1}$. The pDKP hierarchy implies that each component $\phi_{k}, k \in \mathbb{Z}$, satisfies the pKP hierarchy and its remaining content is a special pKP Bäcklund transformation (BT) acting between neighbouring sites on the linear lattice labeled by $k[35,39]$. This suggests a way to extend the method of section 3 to construct exact solutions of the pDKP hierarchy. What is needed is a suitable extension of (2.5) that accounts for the BT and this is offered by proposition 1 .

Theorem 3 Let $\mathbb{A}$ be a WNA algebra with a constant element $\nu \in \mathbb{A} \backslash \mathbb{A}^{\prime}$. Any solution

$$
f=\left(\nu-\phi_{k}\right)_{k \in \mathbb{Z}}
$$

of the hierarchy (2.5) together with the compatible constraint?

$$
f^{+} \circ f=0
$$

yields a solution $\phi=\left(\phi_{k}\right)_{k \in \mathbb{Z}}$ of the $p D K P$ hierarchy in $\mathbb{A}^{\prime}$.

Proof: Since $\left[f^{+}\right]=[f]$, the compatibility follows by setting $f^{\prime}=f^{+}$and $\alpha=0$ in proposition 1 Using $f_{t_{1}}=f \circ f$, we rewrite (2.9) as

$$
\lambda^{-1}\left(f-f_{-[\lambda]}\right)+\left(f-f_{-[\lambda]}\right) \circ f-f_{t_{1}}=0 .
$$

Inserting $f=\nu-\phi$, this takes the form

$$
\lambda^{-1}\left(\phi-\phi_{-[\lambda]}\right)-\phi_{t_{1}}-\left(\phi-\phi_{-[\lambda]}\right) \circ \phi=\theta-\theta_{-[\lambda]}
$$

with $\theta:=-\phi \circ \nu$. Next we use (4.4) and $f_{t_{1}}=f \circ f$ to obtain $\left(f^{+}-f\right) \circ f+f_{t_{1}}=0$, from which we get

$$
\phi_{t_{1}}-\left(\phi^{+}-\phi\right) \circ \phi=\theta^{+}-\theta \text {. }
$$

Together with the previous equation, this leads to

$$
\lambda^{-1}\left(\phi-\phi_{-[\lambda]}\right)-\left(\phi^{+}-\phi_{-[\lambda]}\right) \circ \phi=\theta^{+}-\theta_{-[\lambda]}
$$

(which is actually equivalent to the last two equations), so that

$$
\Omega(\lambda)=\theta^{+}-\theta_{-[\lambda]},
$$

\footnotetext{
${ }^{8}$ This functional representation of the pDKP hierarchy is equivalent to (3.32) in [39].

${ }^{9}$ Note that (4.4) implies $f^{n+} o_{n} f=0$, where $f_{k}^{n+}:=f_{k+n}$. This follows by induction from $f^{(n+1)+} \circ_{n+1} f=$ $f^{(n+1)+} \circ\left(f^{n+} \circ_{n} f\right)-\left(f^{(n+1)+} \circ f^{n+}\right) \circ_{n} f=f^{(n+1)+} \circ\left(f^{n+} \circ_{n} f\right)-\left(f^{+} \circ f\right)^{n+} \circ_{n} f$, where we used (2.3) and $\left[f^{n+}\right]=[f]$ in the first step.
} 
which is easily seen to solve (4.1).

Let us choose the WNA algebra of section 310 Evaluation of (2.5) leads to the matrix Riccati hierarchy (3.5), and (4.4) with $f^{+}=\nu+C-\phi^{+}$becomes

$$
S+C R+(L+C Q) \phi-\phi^{+} R-\phi^{+} Q \phi=0,
$$

which can be rewritten as

$$
\phi^{+}=(S+L \phi)(R+Q \phi)^{-1}+C=Y^{+}\left(X^{+}\right)^{-1}
$$

(assuming that the inverse matrices exist), where $X^{+}, Y^{+}$are the components of

$$
Z^{+}=T H Z=T H e^{\xi(H)} Z^{(0)},
$$

with $Z, H, T$ taken from section 3 . Deviating from the notation of section 3 , we write $Z^{(0)}$ for the constant vector, since $Z_{0}$ should now denote the component of $Z$ at the lattice site 0 . In order that (4.7) defines a pDKP solution on the whole lattice, we need $H$ invertible. Since the matrix $C$, and thus also $T$, may depend on the lattice site $k$, solutions of (4.1) are determined by

$$
Z_{k}=T_{k} H T_{k-1} H \cdots T_{1} H Z_{0}, \quad Z_{-k}=\left(T_{-k} H\right)^{-1}\left(T_{-k+1} H\right)^{-1} \cdots\left(T_{-1} H\right)^{-1} Z_{0}, \quad k \in \mathbb{N}
$$

This corresponds to a sequence of Darboux transformations applied to the matrix pKP solution $\phi_{0}$ determined by $Z_{0}$ (cf. [35]). $\phi_{1}$ is then given by (4.6) in terms of $\phi_{0}$, and

$$
\begin{aligned}
\phi_{2}= & {\left[L S+S R+L C_{1} R+\left(L^{2}+S Q+L C_{1} Q\right) \phi_{0}\right] } \\
& \times\left[R^{2}+Q S+Q C_{1} R+\left(Q L+R Q+Q C_{1} Q\right) \phi_{0}\right]^{-1}+C_{2}
\end{aligned}
$$

shows that the action of the $T_{k}$ becomes considerably more involved for $k>1$. In the special case $T_{k}=I_{N+M}$ (so that $C_{k}=0$ ), we have

$$
Z_{k}=e^{\xi(H)}\left(H^{k} Z_{0}^{(0)}\right) \quad k \in \mathbb{Z} .
$$

If $X_{k}^{(0)}, Y_{k}^{(0)}$ are the components of the vector $H^{k} Z_{0}^{(0)}$, the lattice component $\phi_{k}$ of the pDKP solution determined in this way is therefore just given by the pKP solution of section 3 with initial data $($ at $\mathbf{t}=0$ )

$$
\phi_{k}^{(0)}=Y_{k}^{(0)}\left(X_{k}^{(0)}\right)^{-1}=L^{k} \phi_{0}^{(0)}\left[R^{k}+\left(R^{k} K-K L^{k}\right) \phi_{0}^{(0)}\right]^{-1} .
$$

With the restrictions of example 1 in section 3, assuming that $L$ and $R$ are invertible (so that $H$ is invertible), the corresponding solution of the matrix pDKP hierarchy (in the matrix algebra with product $A \circ B=A(R K-K L) B)$ is

$$
\phi_{k}=e^{\xi(L)} L^{k} \phi_{0}^{(0)}\left[R^{k}\left(I_{N}+K \phi_{0}^{(0)}\right)-e^{-\xi(R)} K e^{\xi(L)} L^{k} \phi_{0}^{(0)}\right]^{-1} e^{-\xi(R)} \quad k \in \mathbb{Z},
$$

which leads to

$$
\varphi_{k}=\left(\log \tau_{k}\right)_{t_{1}} \quad \text { with } \quad \tau_{k}=\operatorname{det}\left(R^{k}\left(I_{N}+K \phi_{0}^{(0)}\right)-e^{-\xi(R)} K e^{\xi(L)} L^{k} \phi_{0}^{(0)}\right) \quad k \in \mathbb{Z} .
$$

If $Q=R K-K L$ has rank 1, this is a solution of the scalar pDKP hierarchy 11 As a special case, let us choose $M=N, L=\operatorname{diag}\left(p_{1}, \ldots, p_{N}\right), R=\operatorname{diag}\left(q_{1}, \ldots, q_{N}\right)$, and $K$ with entries $K_{i j}=\left(q_{i}-p_{j}\right)^{-1} 12$ Then $Q$ has rank 1 and we obtain $N$-soliton tau functions of the scalar discrete KP equation. These can also be obtained with the help of the Birkhoff decomposition method using appropriate initial data as in $[56,57]$.

In case of example 2 of section 3 , the matrix $H$ is not invertible, so that 4.7) does not determine a DKP solution.

\footnotetext{
${ }^{10}$ Since there is only a single element $\nu$, the matrices $L, R, S$ do not depend on the discrete variable $k$.

${ }^{11} \operatorname{Recall}$ that $\varphi=\operatorname{tr}(Q \phi)$ (cf. 3.9) determines a homomorphism if $Q$ has rank 1. As a consequence, if $\phi$ solves the matrix pDKP hierarchy (4.1), then $\varphi$ solves the scalar pDKP equation.

${ }^{12}$ The condition (3.2) requires $q_{i} \neq p_{j}$ for all $i, j=1, \ldots, N$
} 


\section{From WNA to Gelfand-Dickey}

Let $\mathcal{R}$ be the complex algebra of pseudo-differential operators [5]

$$
\mathcal{V}=\sum_{i \ll \infty} v_{i} \partial^{i}
$$

with coefficients $v_{i} \in \mathfrak{A}$, where $\mathfrak{A}$ is the complex differential algebra of polynomials in (in general noncommuting) symbols $u_{n}^{(m)}, m=2,3, \ldots, n=0,1,2, \ldots$, where $\partial\left(u_{n}^{(m)}\right)=u_{n}^{(m+1)}$ and $\partial(v w)=$ $\partial(v) w+v \partial(w)$ for $v, w \in \mathfrak{A}$. We demand that $u_{n}^{(m)}, n=2,3, \ldots, m=0,1,2, \ldots$, are algebraically independent in $\mathfrak{A}$, and we introduce the following linear operators on $\mathcal{R}$,

$$
S(\mathcal{V}):=\mathfrak{L} \mathcal{V}, \quad \pi_{+}(\mathcal{V}):=\mathcal{V}_{\geq 0}, \quad \pi_{-}(\mathcal{V}):=\mathcal{V}_{<0},
$$

where

$$
\mathfrak{L}=\partial+u_{2} \partial^{-1}+u_{3} \partial^{-2}+\cdots .
$$

Here $\mathcal{V}_{\geq 0}$ is the projection of a pseudo-differential operator $\mathcal{V}$ to its differential operator part, and $\mathcal{V}_{<0}:=$ $\mathcal{V}-\mathcal{V}_{\geq 0}$

Let $I$ denote the identity of $\mathcal{R}$ (which we identify with the identity in $\mathfrak{A}$ ), and let $\mathcal{O}$ be the subspace of linear operators on $\mathcal{R}$ spanned by $S$ and elements of the form

$$
S \pi_{ \pm} S \pi_{ \pm} \cdots \pi_{ \pm} S
$$

(with any combination of signs). $\mathcal{O}$ becomes an algebra with the product given by

$$
A \circ B:=A \pi_{+} S \pi_{-} B .
$$

$(\mathcal{O}, \circ)$ is then generated by the elements

$$
\left(S \pi_{-}\right)^{m} S\left(\pi_{+} S\right)^{n} \quad m, n=0,1, \ldots .
$$

Let us furthermore introduce $\mathcal{A}:=\{v \in \mathfrak{A}: v=\operatorname{res}(A(I)), A \in \mathcal{O}\}$, where res takes the residue (the coefficient of $\partial^{-1}$ ) of a pseudo-differential operator. This is a subalgebra of $\mathfrak{A}$, since for $A, B \in \mathcal{O}$ we have

$$
\operatorname{res}(A(I)) \operatorname{res}(B(I))=\operatorname{res}\left(A \pi_{+} S \pi_{-} B(I)\right),
$$

so that the product of elements of $\mathcal{A}$ is again in $\mathcal{A}$. As a consequence of this relation (read from right to left), $\mathcal{A}$ is generated by the elements $\operatorname{res}\left(\left(S \pi_{-}\right)^{m} S\left(\pi_{+} S\right)^{n}(I)\right), m, n=0,1, \ldots$ Based on the following preparations, we will argue that $\mathcal{A}$ and $(\mathcal{O}, \circ)$ are actually isomorphic algebras.

Lemma 1 For all $\mathcal{V} \in \mathcal{R}$,

$$
\operatorname{res}\left(\left(S \pi_{-}\right)^{m} \mathcal{V}\right)=\operatorname{res}\left(\mathcal{D}_{m} \mathcal{V}\right) \quad m=0,1, \ldots,
$$

where $\mathcal{D}_{0}=I$ and $\left\{\mathcal{D}_{m}\right\}_{m=1}^{\infty}$ are the differential operators which are recursively determined by $\mathcal{D}_{m}=$ $\left(\mathcal{D}_{m-1} \mathfrak{L}\right)_{\geq 0}$.

Proof: We do the calculation for $m=2$. This is easily generalized to arbitrary $m \in \mathbb{N}$.

$$
\begin{aligned}
\operatorname{res}\left(\left(S \pi_{-}\right)^{2} \mathcal{V}\right) & =\operatorname{res}\left(\mathfrak{L}\left(\mathfrak{L} \mathcal{V}_{<0}\right)_{<0}\right)=\operatorname{res}\left(\mathfrak{L}_{\geq 0} \mathfrak{L} \mathcal{V}_{<0}\right) \\
& =\operatorname{res}\left(\left(\mathfrak{L}_{\geq 0} \mathfrak{L}\right)_{\geq 0} \mathcal{V}\right)=\operatorname{res}\left(\mathcal{D}_{2} \mathcal{V}\right)
\end{aligned}
$$




\section{Proposition 3}

$$
\operatorname{res}\left(\left(S \pi_{-}\right)^{m} S\left(\pi_{+} S\right)^{n}(I)\right)=\sum_{k=0}^{m}\left(\begin{array}{c}
m \\
k
\end{array}\right) u_{m+n+2-k}^{(k)}+\text { terms nonlinear in } u_{k}^{(j)} \quad m, n=0,1, \ldots
$$

Proof: According to the preceding lemma, we have

$$
\operatorname{res}\left(\left(S \pi_{-}\right)^{m} S\left(\pi_{+} S\right)^{n}(I)\right)=\operatorname{res}\left(\mathcal{D}_{m} S\left(\pi_{+} S\right)^{n}(I)\right) .
$$

Next we note that $\mathcal{D}_{m}=\partial^{m}+D_{m},\left(\pi_{+} S\right)^{n}(I)=\partial^{n}+D_{n}^{\prime}$ with differential operators $D_{m}, D_{n}^{\prime}$ (of degree smaller than $m$, respectively $n$ ) such that each of its summands contains factors from $\left\{u_{k}^{(j)}\right\}$ (so their coefficients are non-constant polynomials in the $\left.u_{k}^{(j)}\right)$. It follows that

$$
\begin{aligned}
\operatorname{res}\left(\left(S \pi_{-}\right)^{m} S\left(\pi_{+} S\right)^{n}(I)\right) & =\operatorname{res}\left(\left(\partial^{m}+D_{m}\right) \mathfrak{L}_{<0}\left(\partial^{n}+D_{n}^{\prime}\right)\right) \\
& =\operatorname{res}\left(\partial^{m} \mathfrak{L}_{<0} \partial^{n}\right)+\text { terms nonlinear in } u_{k}^{(j)} .
\end{aligned}
$$

It remains to evaluate

$$
\begin{aligned}
\operatorname{res}\left(\partial^{m} \mathfrak{L}_{<0} \partial^{n}\right) & =\sum_{j=1}^{\infty} \operatorname{res}\left(\partial^{m} u_{1+j} \partial^{n-j}\right)=\sum_{j=1}^{\infty} \operatorname{res}\left(\sum_{k=0}^{m}\left(\begin{array}{c}
m \\
k
\end{array}\right) u_{1+j}^{(k)} \partial^{m+n-j-k}\right) \\
& =\sum_{k=0}^{m}\left(\begin{array}{c}
m \\
k
\end{array}\right) u_{m+n+2-k}^{(k)} .
\end{aligned}
$$

According to the last proposition, the linear term with the highest derivative 13 in the residue of $\left(S \pi_{-}\right)^{m} S\left(\pi_{+} S\right)^{n}(I)$ is given by $u_{n+2}^{(m)}$. We conclude that the monomials $\left(S \pi_{-}\right)^{m} S\left(\pi_{+} S\right)^{n}, m, n=$ $0,1, \ldots$, are algebraically independent in $(\mathcal{O}, \circ)$, since any algebraic relation among them would induce a corresponding algebraic relation in the set of $u_{n}^{(m)}$, but we assumed the $u_{n}^{(m)}$ to be algebraically independent. Together with (5.5), this implies that $\mathcal{A}$ and $(\mathcal{O}, \circ)$ are isomorphic algebras.

The last result allows us to introduce a WNA structure directly on $\mathcal{A}$ as follows 14 Augmenting $\mathcal{A}$ with $f$ such that, for $\mathcal{V}, \mathcal{W} \in \mathcal{O}(I)$,

$$
\begin{aligned}
f \circ f:=-\operatorname{res}(\mathrm{L}), & f \circ \operatorname{res}(\mathcal{V}):=\operatorname{res}\left(\mathrm{E} \mathcal{V}_{<0}\right), \\
\operatorname{res}(\mathcal{V}) \circ f:=-\operatorname{res}\left(\mathcal{V}_{<0} \mathrm{E}\right), & \operatorname{res}(\mathcal{V}) \circ \operatorname{res}(\mathcal{W}):=\operatorname{res}(\mathcal{V}) \operatorname{res}(\mathcal{W}),
\end{aligned}
$$

indeed defines a WNA algebra $\mathbb{A}=\mathbb{A}(f)$. The relations (5.8) are well-defined since res $(A(I))$ uniquely determines $A \in \mathcal{O}$. By induction we obtain

$$
\begin{aligned}
& f \circ_{n} f=-\operatorname{res}\left(\mathrm{七}^{n}\right), \quad f \circ_{n} \operatorname{res}(\mathcal{V})=\operatorname{res}\left(\leftarrow^{n} \mathcal{V}_{<0}\right), \\
& \operatorname{res}(\mathcal{V}) \circ_{n} f=-\operatorname{res}\left(\mathcal{V}_{<0} \mathbf{\Xi}^{n}\right), \quad \operatorname{res}(\mathcal{V}) \circ_{n} \operatorname{res}(\mathcal{W})=\operatorname{res}\left(\mathcal{V}_{<0} \mathbf{\Xi}^{n} \mathcal{W}_{<0}\right)
\end{aligned}
$$

Let us now assume that the $u_{n}$ are functions depending on variables $t_{1}, t_{2}, \ldots$, and $\partial=\partial_{t_{1}}$. The hierarchy (2.5) of ODEs,

$$
f_{t_{n}}=f \circ_{n} f=-\operatorname{res}\left(\mathrm{七}^{n}\right) \quad n=1,2, \ldots,
$$

\footnotetext{
${ }^{13}$ If $m=0$, the linear term is simply $u_{n+2}$ and thus again "the linear term with the highest derivative".

${ }^{14}$ Note that the corresponding WNA structure for $(\mathcal{O}, \circ)$ resembles that of example 2 in section 3
} 
by use of the WNA structure implies

$$
\begin{aligned}
& \partial_{t_{n}}\left(\operatorname{res}\left(\longleftarrow^{m}\right)\right)=-\partial_{t_{n}}\left(f \circ_{m} f\right)=-f_{t_{n}} \circ_{m} f-f \circ_{m} f_{t_{n}} \\
& =-\left(f \circ_{n} f\right) \circ_{m} f-f \circ_{m}\left(f \circ_{n} f\right)=\operatorname{res}\left(\bigsqcup^{m}\left(\bigsqcup^{n}\right)_{<0}-\left(\bigsqcup^{n}\right)_{\geq 0} \bigsqcup^{m}\right) \text {. } \\
& =\operatorname{res}\left(\left[\left(\iota^{n}\right)_{\geq 0}, 七^{m}\right]\right) \text {. }
\end{aligned}
$$

Since also $\partial_{t_{n}}\left(\operatorname{res}\left(\mathfrak{L}^{m}\right)\right)=\operatorname{res}\left(\left[\left(\mathfrak{L}^{m}\right)_{\geq 0}, \mathfrak{L}^{n}\right]\right)=\partial_{t_{m}}\left(\operatorname{res}\left(\mathfrak{L}^{n}\right)\right)$, we conclude that if we extend $\mathcal{A}$ to $\tilde{\mathcal{A}}$ by adding an element $\phi=\partial^{-1}\left(u_{2}\right)$, then

$$
\phi_{t_{n}}=\operatorname{res}\left(\mathfrak{L}^{n}\right) \quad n=1,2, \ldots .
$$

It follows that $\nu:=f+\phi$ satisfies $\partial_{t_{n}}(\nu)=0, n=1,2, \ldots$, and is therefore constant. (5.12) determines all the $u_{k}$ in terms of the derivatives of $\phi$ (see [58], for example). From (5.12) with $n=2,3$ and (5.11) with $m=n=2$ we recover the pKP equation

$$
\left(4 \phi_{t_{3}}-\phi_{t_{1} t_{1} t_{1}}-6 \phi_{t_{1}}^{2}\right)_{t_{1}}-3 \phi_{t_{2} t_{2}}+6\left[\phi_{t_{1}}, \phi_{t_{2}}\right]=0
$$

in accordance with the general theory. More generally, the equations (5.11) determine the whole pKP hierarchy. They are the residues of

$$
\partial_{t_{n}}\left(\mathfrak{L}^{m}\right)=\left[\left(\mathfrak{L}^{n}\right)_{\geq 0}, \mathfrak{L}^{m}\right] \quad m, n=1,2, \ldots .
$$

This is equivalent to the Gelfand-Dickey (GD) system

$$
\partial_{t_{n}}(\mathfrak{L})=\left[\left(\mathfrak{L}^{n}\right)_{\geq 0}, \mathfrak{L}\right] \quad n=1,2, \ldots
$$

which is a well-known formulation of the KP hierarchy (see [5], for example).

We have thus shown how the Gelfand-Dickey formulation of the KP hierarchy can be recovered in the WNA framework. In fact, for the particular WNA algebra chosen above the hierarchy (2.5) of ODEs is equivalent to the Gelfand-Dickey formulation of the KP hierarchy.

\section{Conclusions}

In this work we have extended our results on the relation between weakly nonassociative (WNA) algebras and solutions of the KP hierarchy to the discrete KP hierarchy. Furthermore, we also established a connection with the Gelfand-Dickey formulation of the KP hierarchy. As a byproduct, in section 5 we obtained a new realization of the free WNA algebra generated by a single element, which also has a realization in terms of quasi-symmetric functions [15].

There is more, however, we have to understand in the WNA framework. In particular this concerns the multi-component KP hierarchy (see [59] and references therein) and its reductions, which include the Davey-Stewartson, two-dimensional Toda lattice and $N$-wave hierarchies. Our hope is that also in these, and perhaps even further cases of hierarchies, the WNA approach leads (perhaps via a Riccati hierarchy) in a quick way to relevant classes of exact solutions.

\section{Appendix: From Riccati to KP with FORM}

The following FORM program [44,45] verifies that any solution of the first three equations of the Riccati hierarchy (3.5) solves the pKP equation in an algebra with product $A \circ B=A Q B$. 


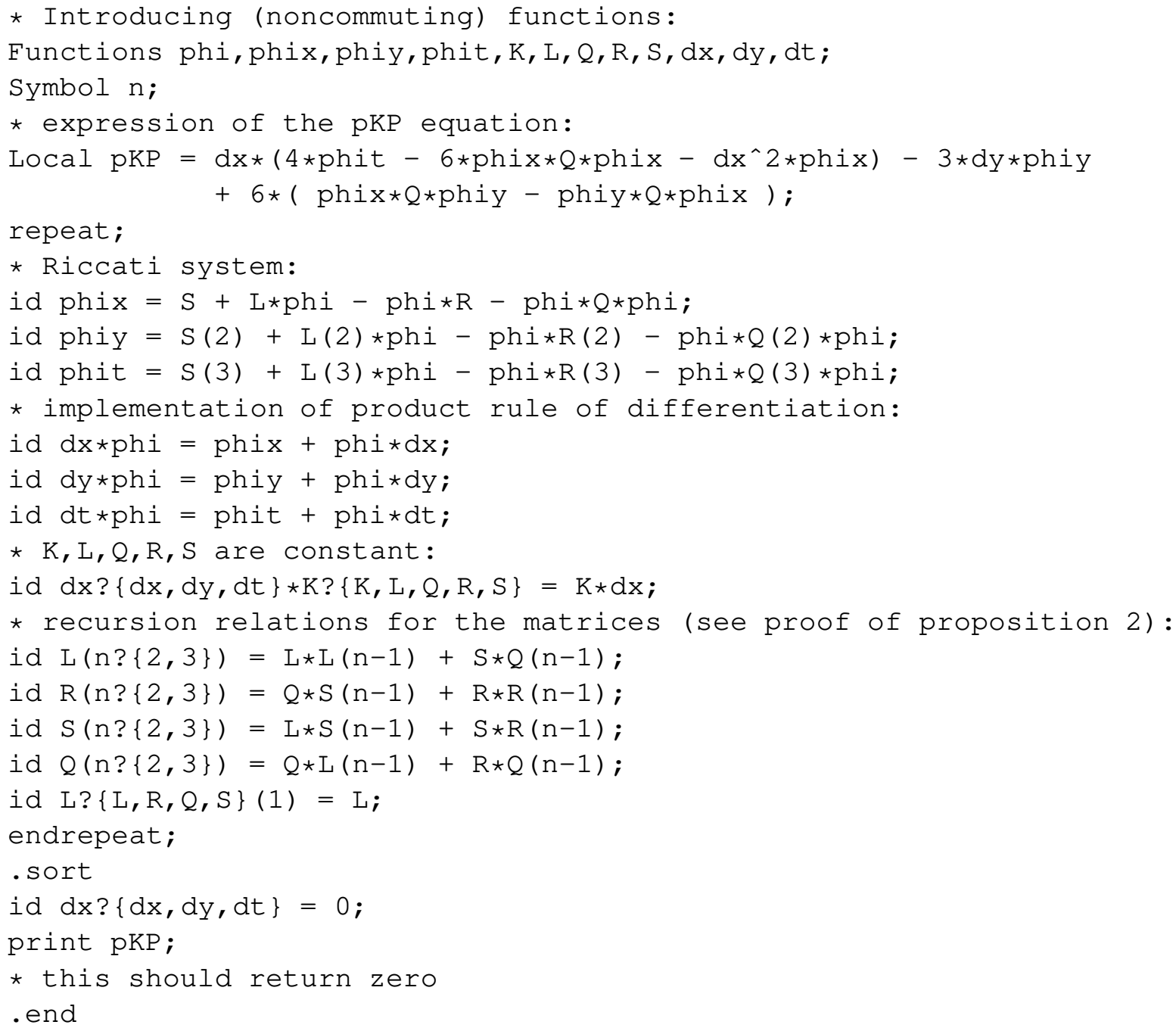

Since the Riccati system can immediately be solved in the case $S=0$ (see section 3), this little program (which further simplifies in the case $S=0$ ) replaces a lengthy calculation by hand and provides an elementary and quick way towards relevant classes of exact solutions of the scalar KP equation, see example 1 .

\section{References}

[1] B.B. Kadomtsev and V.I. Petviashvili. On the stability of solitary waves in a weakly dispersing medium. Sov. Phys. Doklady, 15:539-541, 1970.

[2] E. Infeld and G. Rowlands. Nonlinear Waves, Solitons and Chaos. Cambridge University Press, Cambridge, 2000.

[3] V.B. Matveev and M.A. Salle. Darboux Transformations and Solitons. Springer Series in Nonlinear Dynamics. Springer, Berlin, 1991.

[4] T. Miwa, M. Jimbo, and E. Date. Solitons: Differential Equations, Symmetries and Infinite Dimensional Algebras. Cambridge Univ. Press, Cambridge, 2000.

[5] L.A. Dickey. Soliton Equations and Hamiltonian Systems. World Scientific, Singapore, 2003. 
[6] B.A. Kupershmidt. KP or $m K P$, volume 78 of Mathematical Surveys and Monographs. American Math. Soc., Providence, 2000.

[7] V.M. Buchstaber and I.M. Krichever. Integrable equations, addition theorems, and the RiemannSchottky problem. Russ. Math. Surveys, 61(1):19-78, 2006.

[8] L. Alvarez-Gaumé, C. Gomez, and C. Reina. New methods in string theory. In L. Alvarez-Gaumé, M. B. Green, M. T. Grisaru, R. Iengo, and E. Sezgin, editors, Superstrings '87, pages 341-422, Teaneck, NJ, 1987. World Scientific Publishing.

[9] M. Mulase. KP equations, strings, and the Schottky problem. In M. Kashiwara and T. Kawai, editors, Algebraic Analysis, volume II, pages 473-492, Boston, 1988. Academic Press.

[10] G. Gilbert. The Kadomtsev-Petviashvili equations and fundamental string theory. Commun. Math. Phys., 117:331-348, 1988.

[11] A.Yu. Morozov. String theory: what is it? Sov. Phys. Usp., 35:671-714, 1992.

[12] A. Morozov. Integrability and matrix models. Phys. Uspekhi, 37:1-55, 1994.

[13] M. Mulase. Matrix integrals and integrable systems. In K. Fukaya et. al., editor, Topology, Geometry and Field Theory, pages 111-127, Singapore, 1994. World Scientific.

[14] E. Witten. Algebraic geometry associated with matrix models of two-dimensional gravity. In L.R. Goldberg and A.V. Phillips, editors, Topological methods in modern mathematics (Stony Brook, NY, 1991), pages 235-269. Publish or Perish, Houston, TX, 1993.

[15] A. Dimakis and F. Müller-Hoissen. Nonassociativity and integrable hierarchies. nlin.SI/0601001, 2006.

[16] K. Ihara, M. Kaneko, and D. Zagier. Derivation and double shuffle relations for multiple zeta values. Compositio Math., 142:307-338, 2006.

[17] K. Ihara. Derivations and automorphisms on the algebra of non-commutative power series. Math. J. Okayama Univ., 47:55-63, 2005.

[18] A. Dimakis and F. Müller-Hoissen. An algebraic scheme associated with the noncommutative KP hierarchy and some of its extensions. J. Phys. A: Math. Gen., 38:5453-5505, 2005.

[19] W.T. Reid. Riccati Differential Equations. Academic Press, New York, 1972.

[20] M.Kh. Zakhar-Itkin. The matrix Riccati differential equation and the semi-group of linear fractional transformations. Russ. Math. Surv., 78:89-131, 1973.

[21] H. Abou-Kandil, G. Freiling, V. Ionescu, and G. Jank. Matrix Riccati Equations in Control and Systems Theory. Systems \& Control: Foundations \& Applications. Birkhäuser, Basel, 2003.

[22] L.-L. Chau. Chiral fields, self-dual Yang-Mills fields as integrable systems, and the role of the Kac-Moody algebra. In K. B. Wolf, editor, Nonlinear Phenomena, volume 189 of Lecture Notes in Physics, pages 110-127, Berlin, 1983. Springer.

[23] J. Harnad, Y. Saint-Aubin, and S. Shnider. The soliton correlation matrix and the reduction problem for integrable systems. Commun. Math. Phys., 93:33-56, 1984. 
[24] K. Takasaki. A new approach to the self-dual Yang-Mills equations II. Saitama Math. J., 3:11-40, 1985.

[25] A.K. Common and D.E. Roberts. Solutions of the Riccati equation and their relation to the Toda lattice. J. Phys. A: Math. Gen., 19:1889-1898, 1986.

[26] J. Dorfmeister, E. Neher, and J. Szmigielski. Automorphisms of Banach manifolds associated with the KP-equation. Quart. J. Math. Oxford, 40:161-195, 1989.

[27] A.M. Grundlach and D. Levi. Higher order Riccati equations as Bäcklund transformations. preprint CRM-2469, 1997.

[28] L.A. Ferreira, J.F. Gomes, A.V. Razumov, M.V. Saveliev, and A.H. Zimerman. Riccati-type equations, generalized WZNW equations, and multidimensional Toda systems. Commun. Math. Phys., 203:649-666, 1999.

[29] M. Hazewinkel. Ricatti and soliton equations. In K. Ito and T. Hida, editors, Gaussian Random Fields: The Third Nagoya Levy Seminar, volume 1 of Series on Probability \& Statistics, pages 187-196, Singapore, 1991. World Scientific.

[30] G. Haak. Negative flows of the potential KP-hierarchy. Trans. Amer. Math. Soc., 348:375-390, 1996.

[31] G. Falqui, F. Magri, and M. Pedroni. Bihamiltonian geometry, Darboux coverings, and linearization of the KP hierarchy. Commun. Math. Phys., 197:303-324, 1998.

[32] K. Takasaki. Geometry of universal Grassmann manifold from algebraic point of view. Rev. Math. Phys., 1:1-46, 1989.

[33] G. Falqui, F. Magri, M. Pedroni, and J.P. Zubelli. An elementary approach to the polynomial tau-functions of the KP hierarchy. Theor. Math. Phys., 122:23-26, 2000.

[34] M. Zelikin. Geometry of the cross-ratio and KP-hierarchy. Institut Mittag-Leffler report no. 14, 2002/2003, 2003.

[35] M. Adler and P. van Moerbeke. Vertex operator solutions of the discrete KP-hierarchy. Commun. Math. Phys., 203:185-210, 1999.

[36] L.A. Dickey. Modified KP and discrete KP. Lett. Math. Phys., 48:277-289, 1999.

[37] L. Haine and P. Iliev. Commuting rings of difference operators and an adelic flag manifold. Int. Math. Res. Notices, (6):281-323, 2000.

[38] R. Felipe and F. Ongay. Algebraic aspects of the discrete KP hierarchy. Linear Algebra and its Applications, 338:1-18, 2001.

[39] A. Dimakis and F. Müller-Hoissen. Functional representations of integrable hierarchies. J. Phys. A: Math. Gen., 39:9169-9186, 2006.

[40] H.O. Pflugfelder. Quasigroups and Loops, volume 7 of Sigma Series in Pure Mathematics. Heldermann, Berlin, 1990.

[41] L.V. Bogdanov and B.G. Konopelchenko. Analytic-bilinear approach to integrable hierarchies. II. Multicomponent KP and 2D Toda lattice hierarchies. J. Math. Phys., 39:4701-4728, 1998. 
[42] L.V. Bogdanov. Analytic-Bilinear Approach to Integrable Hierarchies, volume 493 of Mathematics and its Applications. Kluwer, Dordrecht, 1999.

[43] A. Dimakis and F. Müller-Hoissen. Burgers and KP hierarchies: A functional representation approach. to appear in Theor. Math. Phys., 2007.

[44] A. Heck. FORM for Pedestrians. NIKHEF, Amsterdam, 2000.

[45] J.A.M. Vermaseren. FORM Reference Manual. NIKHEF, Amsterdam, 2002.

[46] M. Gekhtman and A. Kasman. On KP generators and the geometry of the HBDE. J. Geom. Phys., 56:282-309, 2006.

[47] V.A. Marchenko. Nonlinear Equations and Operator Algebras. Mathematics and Its Applications. Reidel, Dordrecht, 1988.

[48] H. Aden and B. Carl. On realizations of solutions of the KdV equation by determinants on operator ideals. J. Math. Phys., 37:1833-1857, 1996.

[49] B. Carl and C. Schiebold. Nonlinear equations in soliton physics and operator ideals. Nonlinearity, 12:333-364, 1999.

[50] H. Blohm. Solution of nonlinear equations by trace methods. Nonlinearity, 13:1925-1964, 2000.

[51] B. Carl and C. Schiebold. Ein direkter Ansatz zur Untersuchung von Solitonengleichungen. Jber. Dt. Math.-Verein., 102:102-148, 2000.

[52] W. Han and Y. Li. Remarks on the solutions of the Kadomtsev-Petviashvili equation. Phys. Lett. A, 283:185-194, 2001.

[53] S.-Z. Huang. An operator method for finding exact solutions to vector Korteweg-deVries equations. J. Math. Phys., 44:1357-1388, 2003.

[54] C. Schiebold. From the non-Abelian to the scalar two-dimensional Toda lattice. Glasgow Math. J., 47A:177-189, 2005.

[55] A. Dimakis and F. Müller-Hoissen. From nonassociativity to solutions of the KP hierarchy. Czech. J. Phys., 56:1123-1130, 2006.

[56] K. Ueno and K. Takasaki. Toda lattice hierarchy. In K. Okamoto, editor, Group Representations and Systems of Differential Equations, volume 4 of Advanced Studies in Pure Mathematics, pages 1-95, Amsterdam, 1984. North-Holland.

[57] M. Sakakibara. Factorization methods for noncommutative KP and Toda hierarchies. J. Phys. A: Math. Gen., 37:L599-L604, 2004.

[58] A. Dimakis and F. Müller-Hoissen. Explorations of the extended ncKP hierarchy. J. Phys. A: Math. Gen., 37:10899-10930, 2004.

[59] V.G. Kac and J.W. van der Leur. The $n$-component KP hierarchy and representation theory. $J$. Math. Phys., 44:3245-3293, 2003. 